\title{
Effects of Combustion Regimes on Localized Forced Ignition of Turbulent Stratified Mixture
}

\author{
Kathan Modi ${ }^{1}$, Hitha Uchil ${ }^{1}$, Dipal Patel ${ }^{1}$ \\ ${ }^{1}$ University of Ontario Institute of Technology \\ 2000 Simcoe St N, Oshawa, ON L1H 7K4, Canada \\ kathan.modi@uoit.net; hitha.uchil@uoit.net; dipal.patel@uoit.ca
}

\begin{abstract}
Localized forced ignition of turbulent stratified mixtures $\left(\langle\phi\rangle=1, \phi^{\prime}=0.2\right)$ with different values of mixture inhomogeneity has been analyzed based on Direct Numerical Simulations (DNS) for different values of Karlovitz number $(\mathrm{Ka})$ corresponding to the premixed turbulent combustion regime diagram. The initial values of turbulent fluctuations (i.e. $\left.u^{\prime} / S_{b(\phi=1)}\right)$ and the integral length scale of turbulence (i.e. $L_{11} / l_{f}$ ) have been modified to bring about the change in $K a$. The localized ignition is accounted by a source term in the energy transport equation which deposits energy over a specified time interval. It has been found that combustion takes place predominantly under a premixed mode of combustion following successful ignition. The percentage of heat release due to a premixed mode of combustion increases with increasing $K a$ due to high mixing rate. An increase in $K a$ has been shown to have adverse effects on the burned gas mass. The different level of mixture inhomogeneity shown to have favorable effect with increasing $\mathrm{Ka}$ for sustaining combustion. Furthermore, a stratified combustion mixture has been found to be a more favorable choice over homogeneous mixtures for a given turbulent flow condition for sustaining combustion.
\end{abstract}

Keywords: Stratified mixture; direct numerical simulation; localized forced ignition; equivalence ratio; Karlovitz number.

\section{Introduction}

Localized forced ignition of an inhomogeneous mixture in form of spark or laser plays an important role in the design of efficient Spark Ignition (SI) and Direct Ignition (DI) engines [1]. Forced Ignition in different combustion modes has been subject to numerous analyses. A number of previous analyses concentrated on localized forced ignition of inhomogeneous mixtures where the mixture inhomogeneity is characterized by a gradient of mean equivalence ratio [5]. The mixture inhomogeneity can potentially increase the extent of flame wrinkling and may significantly affect the cycle-to-cycle variation of heat release $[2,3]$. The flame-turbulence interaction in turbulent premixed combustion is often characterized in terms of a non-dimensional number known as Karlovitz number $K a=\left[u^{\prime} / S_{b(\phi=1)}\right]^{3 / 2} / \sqrt{L_{11} / l_{f}}$ (where $L_{11}$ and $l_{f}$ is the longitudinal integral length scale and Zel'dovich flame thickness respectively). A combustion regime often refers to the outcome of competitions between length, time and velocity scales, which characterize turbulence, chemistry, and their coupling. On the regime diagram of premixed turbulent combustion [4] the condition $100>K a>1$ corresponds to thin reaction zone regime, the line $u^{\prime} / S_{b(\phi)}=1$, separates the wrinkled flamelets from the corrugated flamelets, and the line denoted by $K a=100$, separates the thin reaction zones from the broken reaction zones. The line $R e_{t}=1\left(R e_{t}\right.$ is the turbulent Reynolds Number, $R e_{t} \sim u^{\prime} L_{11} / v$, where $v$ is the viscosity) separates all the turbulent flame regimes characterized by $R e_{t}>1$ from the laminar flame regime $\left(R e_{t}<1\right)$. Here, the length scale of mixture inhomogeneity is defined using Taylor micro-scale as $l_{\phi}=\sqrt{6\left\langle[\phi-\langle\phi\rangle]^{2}\right\rangle /\langle\nabla[\phi-\langle\phi\rangle] . \nabla[\phi-\langle\phi\rangle]\rangle}$ where angle bracket indicates the global mean evaluated over the whole computational domain [5]. Many previous analyses focused on various aspects of localized forced ignition of both homogenous and inhomogeneous mixture [1], but the effects of $K a$ on localized forced ignition of a stratified combustible mixture are yet to be analyzed using DNS data $[1-3,5]$. The availability of detailed experimental data for model development and validation is very limited, and the state of modeling for stratified combustion is less advanced than that for non-premixed or fully premixed combustion. This gap has been addressed here by analyzing the influences of different regimes on localized forced ignition of both homogenous and inhomogeneous combustible mixtures using three-dimensional DNS data. 
Table 1: Nomenclature.

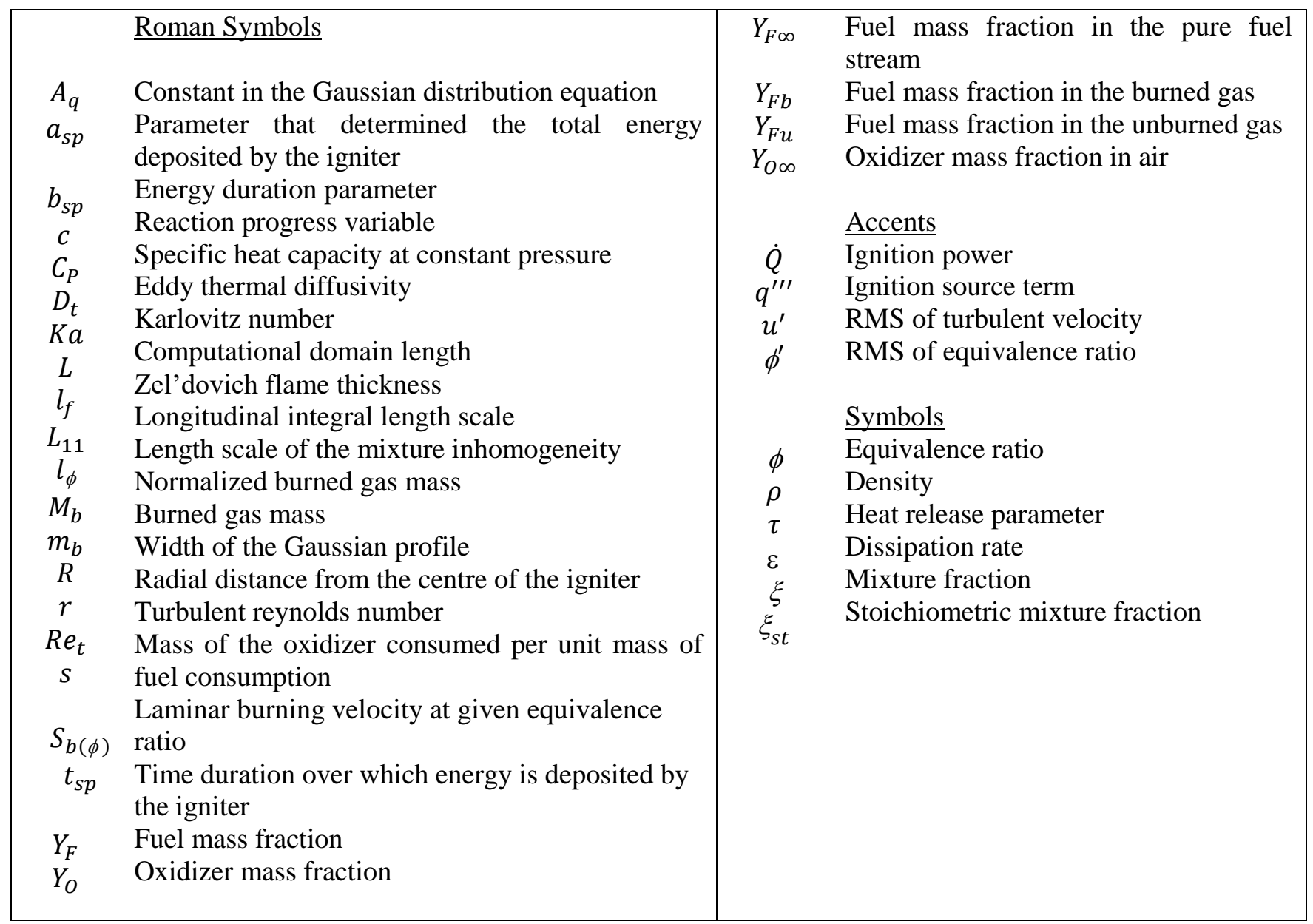

\section{Mathematical Background and Numerical Implementation}

The chemical mechanism is simplified here by single step chemical reaction [6], which takes the form of (Fuel + s Oxidizer $\rightarrow(1+s)$ products), where $s$ indicates the mass of the oxidizer consumed per unit mass of fuel consumption under stoichiometric conditions. The equivalence ratio is defined as $\phi=\frac{F A R}{(F A R)_{s t}}$, where $F A R$ and $F A R_{s t}$ are the actual and stoichiometric fuel- air ratios respectively. The mixture fraction is defined as, $\xi=\left[Y_{F}-\frac{Y_{O}}{S}+\frac{Y_{O \infty}}{s}\right] /\left[Y_{F \infty}+\right.$ $\left.\frac{Y_{O \infty}}{S}\right]$ where $Y_{F}$ and $Y_{O}$ are the local fuel and oxidizer mass fractions respectively, and the stoichiometric mixture fraction is, $\xi_{s t}=Y_{O \infty} /\left[s Y_{F \infty}+Y_{O \infty} / s\right]$ based on this, equivalence ratio is given as $\phi=\left[1-\xi_{s t}\right] \xi /[1-\xi] \xi_{s t}$ where $Y_{F \infty}$ and $Y_{O \infty}$ are the fuel and oxidizer mass fractions in pure fuel and air stream respectively. The extent of the completion of the chemical reaction is quantified using a reaction progress variable, $c=\left(Y_{F u}-Y_{F}\right) /\left(Y_{F u}-Y_{F b}\right)\left(\right.$ or $c=\left[\xi Y_{F \infty}-Y_{F}\right] /\left[\xi Y_{F \infty}-\right.$ $\left.\left.\max \left[0, \frac{\left(\xi-\xi_{s t}\right)}{\left(1-\xi_{s t}\right)}\right] Y_{F \infty}\right]\right)[5,7]$ where $Y_{F u}$ and $Y_{F b}$ are the fuel mass fractions in the unburned and burned gases respectively. According to this $c$ rises from 0 in the fully unburned reactants to 1.0 in the fully burned products.

The localized ignition is modelled by adding a source term $q^{\prime \prime \prime}$ in the energy transport equation which is assumed to follow a Gaussian distribution in the radial direction away from the center of the ignitor and is expressed as: $q^{\prime \prime \prime}(r)=$ $A_{q} \cdot \exp \left(-\frac{r^{2}}{2 R^{2}}\right)$ where $r$ is the radial direction from the center of the ignitor, $R$ is the width of the Gaussian profile and the 
constant $A_{q}$ can be determined by following volume integration as, $\dot{Q}=\int_{v} q^{\prime \prime \prime} d V$, where $\dot{Q}$ is the ignition power, which is defined as $[5,7,8]$ :

$$
\dot{Q}=a_{s p} \rho_{0} C_{P} \tau T_{O}\left(4 \pi l_{f}^{3} / 3\right) \frac{\left[H(t)-H\left(t-t_{s p}\right)\right]}{t_{s p}}
$$

Where $\tau=3.0$ is the heat release parameter. The parameter $a_{s p}$ determines the total energy deposited by the ignitor and is taken in manner where it ensures successful ignition for all the case considered here. Two Heaviside functions $H(t)$ and $H\left(t-t_{s p}\right)$ ensure the ignition source term remains operational for the energy deposition time during the duration $t_{s p}$. The parameter $t_{s p}$ can be expressed as $t_{s p}=b_{s p} t_{f}$, where $b_{s p}$ is the energy deposition duration parameter and $t_{f}=l_{f} / S_{b(\phi=1)}$ is the characteristic chemical time scale. For the present analysis $b_{s p}=0.2$ and $R \approx 1.55 l_{f}$ are taken following previous studies $[5,7,8]$ to ensure successful ignition and Lewis number of all species is taken to be unity. The pseudo-spectral method proposed by Eswaran and Pope was used for generating initial $\phi$ distribution following bi-modal distribution [9].

Table 2: Simulation Table.

\begin{tabular}{|c|c|c|c|c|}
\hline & $\begin{array}{c}\text { Stratified } \\
\text { mixture } \\
\langle\phi\rangle=1 ; \\
\phi^{\prime}=0.2\end{array}$ & & & $\begin{array}{c}\text { Homogenous } \\
\text { mixture }\end{array}$ \\
\hline$K a$ & $\frac{L_{11}}{l_{f}}=10$ & $\frac{l_{\phi}}{l_{f}}=1.6$ & $\frac{l_{\phi}}{l_{f}}=4.5$ & \\
\hline 0.052 & $\frac{u^{\prime}}{S_{b(\phi=1)}}=0.8$ & WFA & WFC & HWF \\
\hline 0.226 & $\frac{u^{\prime}}{S_{b(\phi=1)}}=1.5$ & CFA & CFC & HCF \\
\hline 10 & $\frac{u^{\prime}}{S_{b(\phi=1)}}=10$ & 10TRA & 10TRC & H10TR \\
\hline 37.2 & $\frac{u^{\prime}}{S_{b(\phi=1)}}=24$ & 24TRA & 24TRC & H24TR \\
\hline
\end{tabular}

A compressible three-dimensional DNS code SENGA [10] was used to carry out the simulations under decaying turbulence in a domain of size $51 l_{f} \times 51 l_{f} \times 51 l_{f}$, which further discretized by a Cartesian grid of size $310 \times 310 \times 310$ with uniform grid spacing. The boundaries in the $x_{1}$ - direction are taken to be partially non-reflecting (using Navier-Stokes Characteristic Boundary Conditions), and all other boundaries are periodic [11]. A standard pseudo-spectral method [12] is used to initialize the turbulent velocity fluctuations. The simulation parameters are listed in Table 1 . The case names are chosen in such manner so that [WF] stands for Wrinkled Flamelet; [CF] stands for Corrugated Flamelet; [TR] stands for Thin Reaction of the regime, $[\mathrm{A}]$ and $[\mathrm{C}]$ indicate increasing values of $l_{\phi} / l_{f}$. 

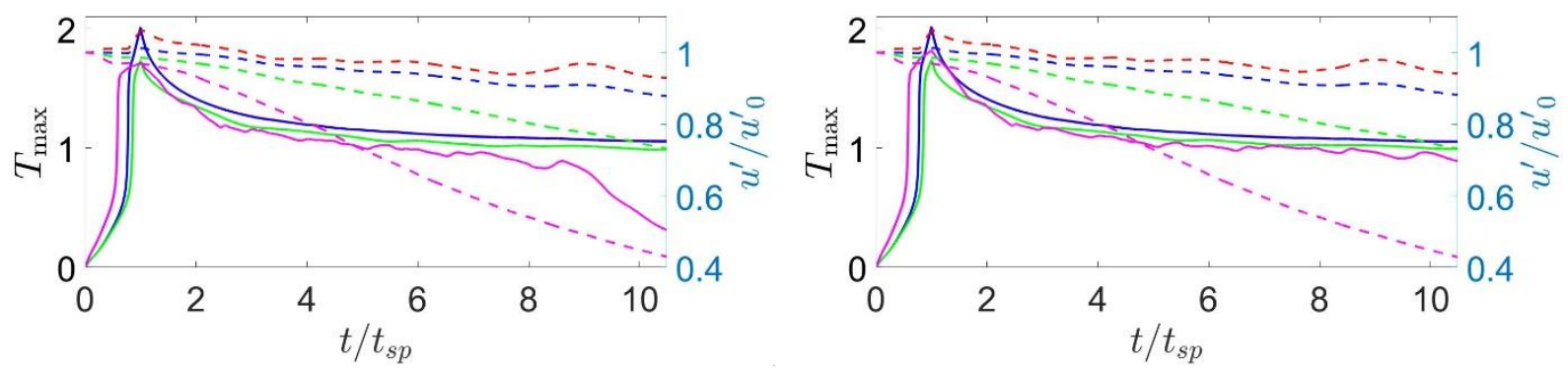

$$
K a=0.052, K a=0.226, K a=10, K a=37.2
$$

Fig. 1(a): Temporal evolution of $\mathrm{T}_{\max }$ (solid line) and corresponding turbulence decay profile (broken line) for all the cases showing $l_{\phi} / l_{f}=1.6$ (left) and $l_{\phi} / l_{f}=4.5$ (right).
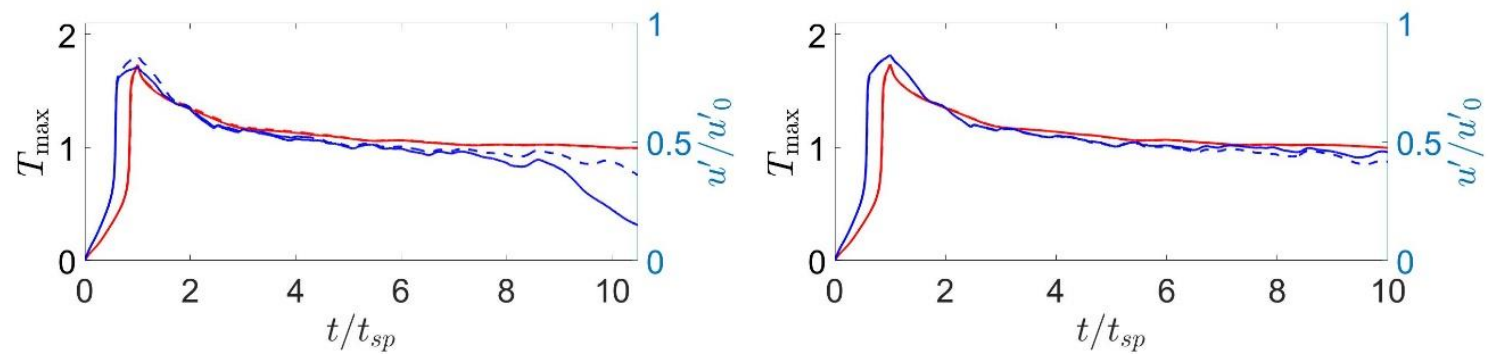

Fig. 1(b): Temporal evolution of $\mathrm{T}_{\max }$ and corresponding turbulence decay profile for the stratified (solid line) and homogenous cases

$$
\text { (broken line) } \frac{u^{\prime}}{s_{b(\phi=1)}}=10 \text { and } \frac{u^{\prime}}{S_{b(\phi=1)}}=24 \text { showing } l_{\phi} / l_{f}=1.6 \text { (left) and } l_{\phi} / l_{f}=4.5 \text { (right). }
$$

\section{Results and Discussion}

\subsection{Temporal evolution of Non-dimensional maximum temperature $\left(T_{\max }\right)$}

The temporal evolutions of the maximum values of non-dimensional temperature (where $T_{\max }=\left[\hat{T}_{\max }-T_{0}\right] /\left[T_{a d(\phi=1)}-T_{0}\right]$ ) and corresponding turbulence decay profile for all the cases from Table 1 are shown in fig 1 . Figure 1(a) \& (b) shows that $\mathrm{T}_{\max }$ rises with time until $t=1 t_{s p}$ due to energy deposition during $0<t<t_{s p}$, showing that the $\mathrm{T}_{\max }$ attains its peak value during the energy deposition. The high thermal gradient between the hot gas and the surrounding unburned gas gives rise to a high rate of heat transfer from the ignition kernel. This, in turn, leads to a decrease in $T_{\max }$. Figure 1(a) shows that with increasing $K a$, for $u^{\prime} / S_{b(\phi=1)}=24$ the peak value of $\mathrm{T}_{\max }$ at $t=t_{s p}$, reduces for a given amount of energy deposition. In fig 1(a) it is been observed that all the stratified cases attain self-sustain combustion but except for the case 24TRA where it follows the successful ignition but failed to attain the sustainable combustion. Figure 1(b) shows the comparison between the homogenous and the stratified cases, 24TR, and 10TR in which the homogenous case (H24TR) accomplishes selfsustained combustion following successful ignition, whereas the stratified mixture (24TRA) fails to sustain combustion following successful ignition. Self-sustained combustion is observed for the higher $l_{\phi} / l_{f}$ (i e $l_{\phi} / l_{f}=4.5$ ). Moreover, it can be seen from fig 1 that high value of temperature during ignition can be avoided for higher values of $K a$, using a stratified mixture with high $l_{\phi} / l_{f}$ which ultimately reduces thermal $N O_{x}$ emission and still achieve self-sustained combustion [13].

Furthermore, in this DNS configuration, it can be seen that $u^{\prime}$ decays for all the cases especially for high $\mathrm{Ka}$ values with time as the eddy thermal diffusivity $D_{t} \sim u^{\prime} L_{11}[8]$ decreases with time. The surface area to volume ratio of the hot gas kernel decreases with the expansion of the flame kernel. This along with decreasing surface to volume ratio with time leads to a reduction in the magnitude of heat transfer rate from the hot gas kernel as time progresses, which leads to a more favorable condition for thermal runaway in the cases of high values of the $\mathrm{T}_{\max }$. Therefore the duration over which the peak value of $\mathrm{T}_{\max }$ is obtained after $t=t_{s p}$ also depends on the background fluid turbulence. 


\subsection{Spatial distributions of fuel mass fraction $\left(Y_{F}\right)$ and equivalence ratio $(\phi)$}

The distributions of fuel mass fraction $\left(Y_{F}\right)$ and equivalence ratio $(\phi)$ at the different time intervals for the selected cases are shown in fig 2(a). Figure 2(a) shows that the fuel mass fraction $Y_{F}$ is depleted at the regions associated with high values of $T$ due to consumption of fuel as a result of chemical reaction. The reaction rate is almost negligible for case 24TRA at the time $t=10.5 t_{s p}$, as this case failed to sustain combustion, following successful ignition after $t>8 t_{s p}$ (fig not shown). Additionally, it is seen that at the high-temperature region, where the chemical reaction takes place, has been fragmented in the case 24TRA due to penetration of energetic eddies into the flame characteristic of thin reaction regime. This fragmented high-temperature region is indicative of a disturbance of the reaction zone by turbulence under high values of $K a$.
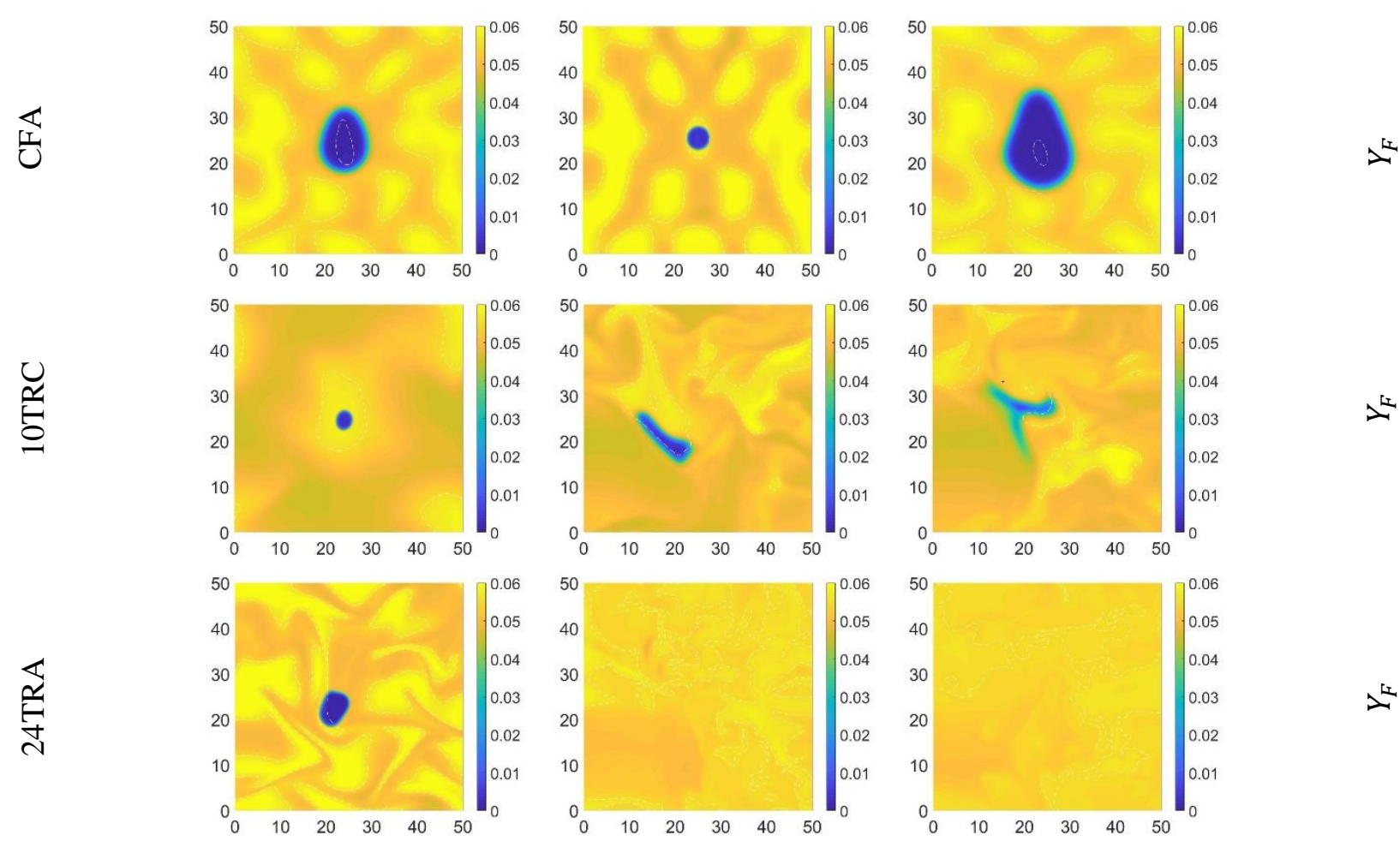

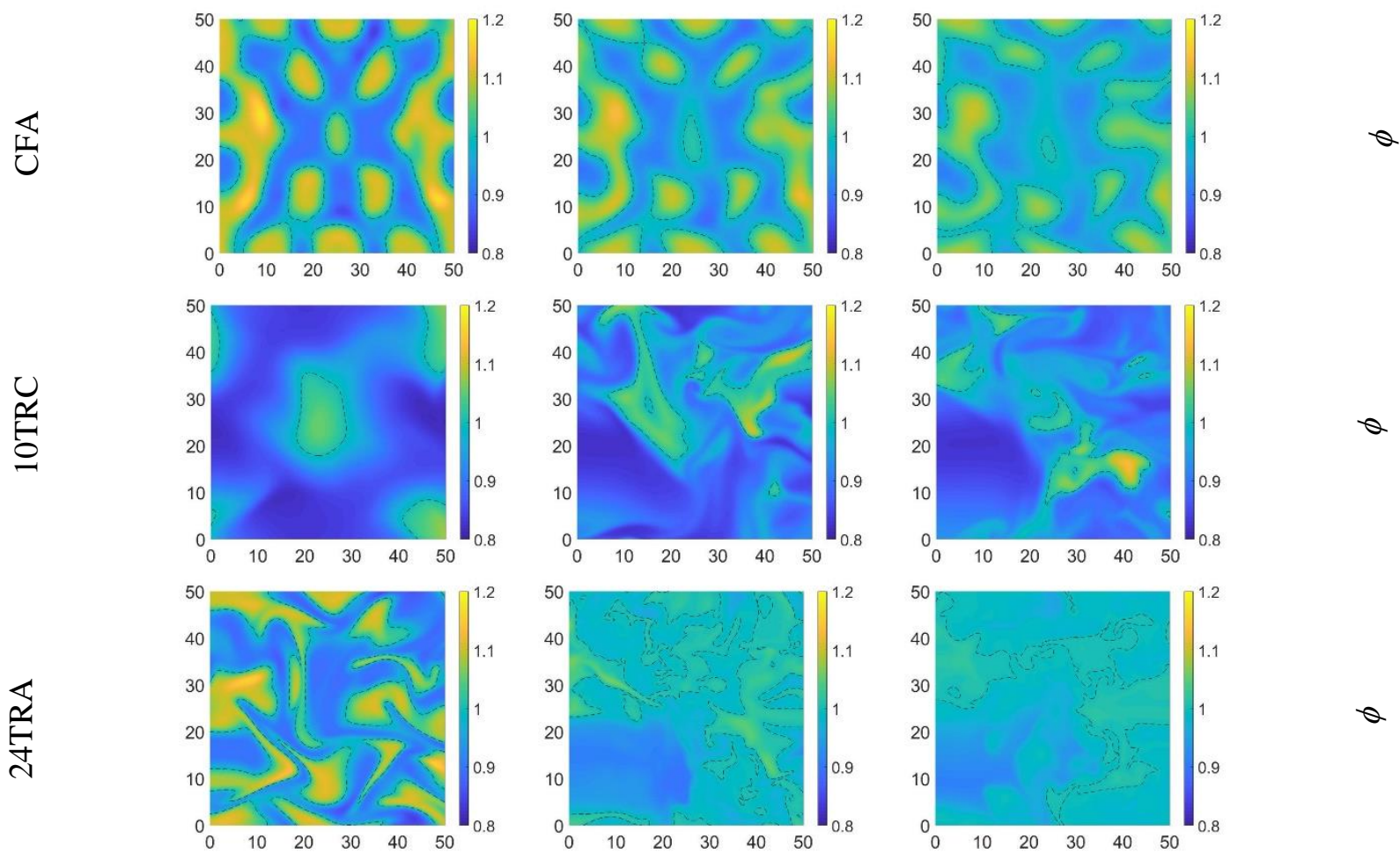

Fig. 2(a): Distribution of $Y_{F}\left(1^{\text {st }}, 2^{\text {nd }} \& 3^{\text {rd }}\right.$ row $)$ and $\phi\left(4^{\text {th }}, 5^{\text {th }} \& 6^{\text {th }}\right.$ row $)$ for the cases CFA, 10TRC $\& 24$ TRA [showing $\mathrm{t}=1.05 t_{s p}$ $\left(1^{\text {st }}\right.$ column $), \mathrm{t}=5.78 t_{s p}\left(2^{\text {nd }}\right.$ column $) \& \mathrm{t}=10.5 t_{s p}\left(3^{\text {rd }}\right.$ column $\left.)\right]$.

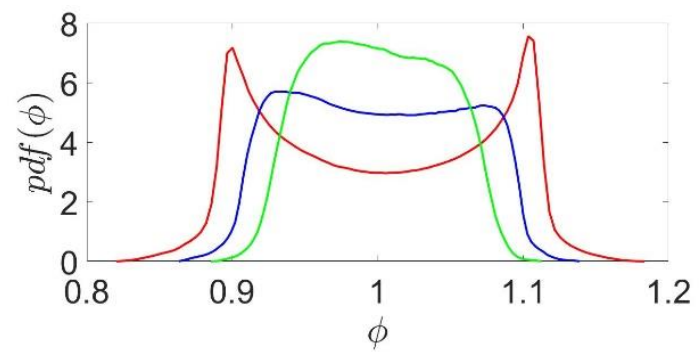

WFA

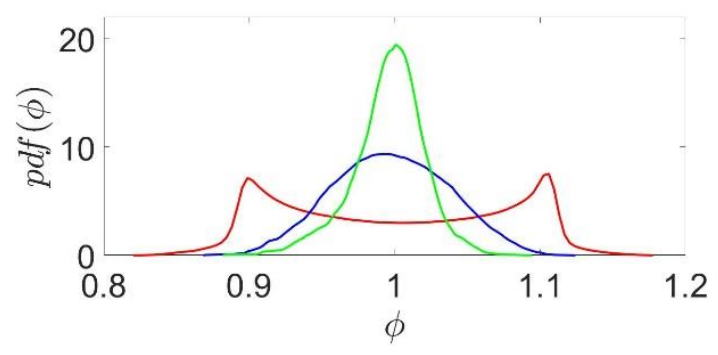

10TRA

Fig. 2(b): Temporal evolution of the $p d f$ of $\phi$ for the cases WFA, $l_{\phi} / l_{f}=1.6$ (left) and 10TRA, $l_{\phi} / l_{f}=4.5$ (right) at $\mathrm{t}=1.05 t_{s p}$,

$$
\mathrm{t}=5.78 t_{s p}, \mathrm{t}=10.5 t_{s p} \text {. }
$$

The evolution of mixing process can be illustrated from analyzing the temporal evolution of $p d f$ of equivalence ratio $(\phi)$, shown in the fig 2(b). Peak values of $p d f \mathrm{~s}(\phi)$ at $\phi \approx\langle\phi\rangle=1$ can be seen for the case 10TRA over the period of time. Figure $2(\mathrm{~b})$ shows that the width of the $p d f \mathrm{~s}$ of $(\phi)$ decreases with time suggesting that the level of non-uniformity in the $\phi$ distribution decreases as time progresses for the cases WFA and 10TRA.

\subsection{Extent of Burning}

Since fig. 1 provides the information on successful ignition, it is necessary to assess the temporal evolution of burned gas mass with time. The extent of burning can be characterized by the burned mass $m_{b}$ with $c \geq 0.9$. The temporal evolution of the burned gas mass normalized with the mass of an unburned gas sphere with a radius equal to $l_{f}$ (i.e. $M_{b}=$ $\left.\left[m_{b(c \geq 0.9)}\right] /\left[(4 / 3) \pi \rho_{o} l_{f}^{3}\right]\right)[5,7,8]$ for all the cases listed in Table are shown in the fig 3 . Higher values of $M_{b}$ is attained 
for the cases WF and CF as compare to TR, because of eddy thermal diffusivity $D_{t} \sim u^{\prime} L_{11}$, thereby chemical heat release overcomes the heat transfer for lower value of $K a$ number. Figure 3 reveals that $M_{b}$ in the case 24TRA does not rise with time as compared to other. This is due to the heat transfer from hot gas kernel beating the chemical heat release at early phases of flame development, which results in reduction of $M_{b}$ with time.
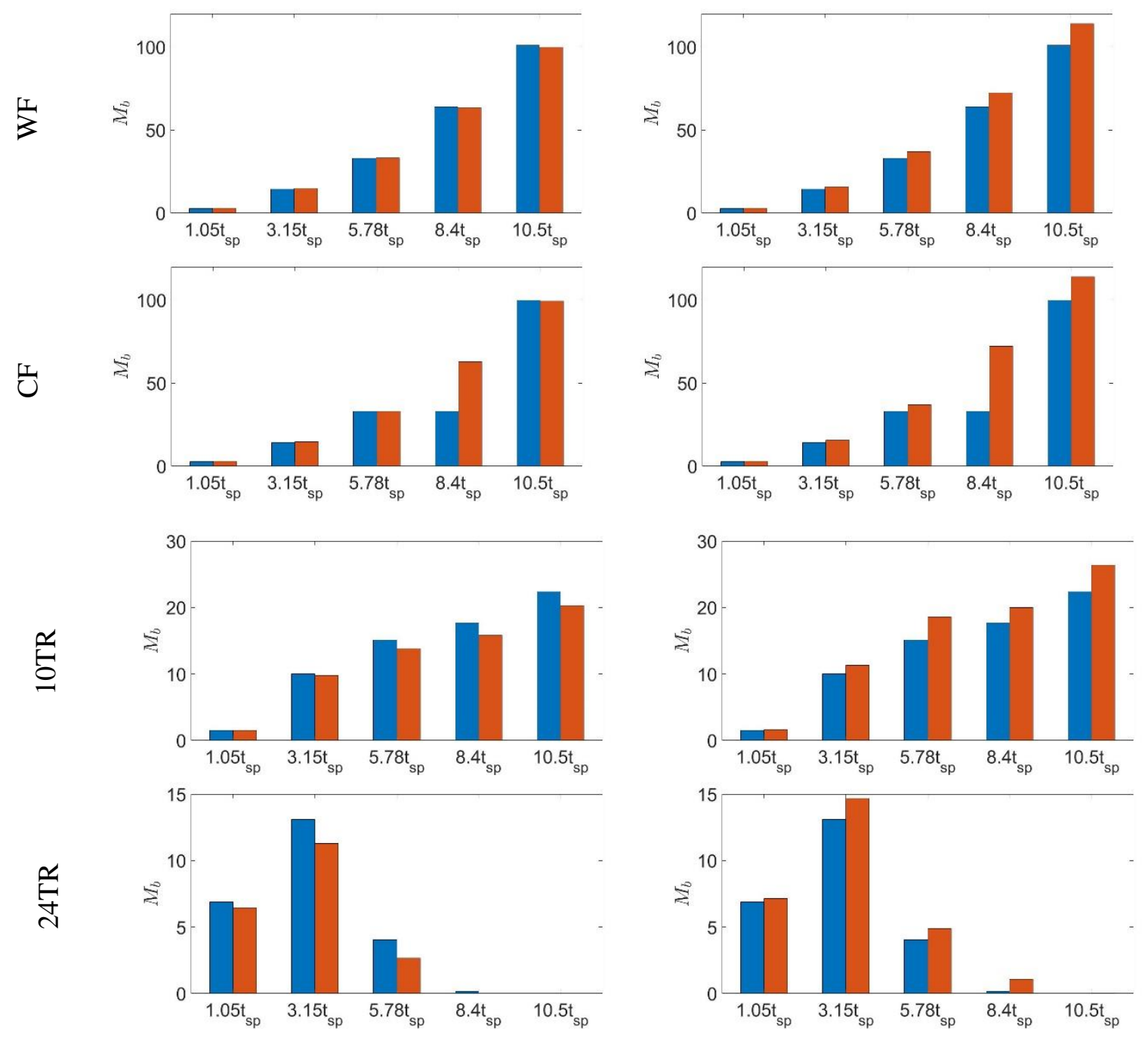

Fig. 3: Temporal evolution of $\mathrm{M}_{\mathrm{b}}$ for all the homogenous and stratified cases from Table $1, l_{\phi} / l_{f}=1.6$ (left) and $l_{\phi} / l_{f}=4.5$ (right).

The probability of finding high values of $c$ decrease with increasing $u^{\prime}$ due to the augmentation of the heat transfer rate from the hot gas kernel. For the cases 24TR, after $t>8 t_{s p}$, when the heat loss overcomes chemical heat release, the hot gas kernel shrinks and the heat release drops drastically once the probability of finding the most reactive $c$ isosurface disappears, which eventually leads to flame extinction. Also, the fig 3 reveals that it is possible to achieve higher value of $M_{b}$ in the stratified mixture compare to the corresponding homogeneous mixture case for higher value of $l_{\phi} / l_{f}$ and given realization of $\phi$ distribution. The reduction in burning rate with high value of $u^{\prime}$ in stratified mixture is found to be consistent with previous experimental [14] and computational [5] findings. 


\section{Conclusions}

The effects of different Karlovitz number $(\mathrm{Ka})$ for successful ignition and self-sustained combustion has been investigated using DNS configuration. The condition, which led to self-sustained flame propagation following successful ignition, depend on both distribution mixture and $\mathrm{Ka}$. Self-sustained combustion has been obtained for all the cases except for the case 24TRA, $l_{\phi} / l_{f}=1.6$. The detrimental effects of high values of $u^{\prime}$ are more critical for high values of $K a$. The increase in heat transfer rate from the hot gas kernel with an increase in $u^{\prime}$ leads to a decrease in the extent of burning irrespective of the values of $l_{\phi} / l_{f}$. It is possible to obtain a higher value for the extent of burning in the stratified mixture compare to corresponding homogeneous mixture even for a higher value of $K a$. Additionally, increase in $u^{\prime}$ decreases extent of burning and may lead to misfire for larger values of $u^{\prime}$. However, the qualitative nature of flame propagation following localized forced ignition is not likely to change in the presence of detailed chemical kinetics, three-dimensional DNS study with detailed chemistry and transport will be required to achieve more comprehensive physical understanding and accurate qualitative predictions.

\section{Acknowledgments}

The authors are grateful to Compute Canada for providing computational support.

\section{References}

[1] E. Mastorakos, "Ignition of turbulent non-premixed flames," Progress in Energy and Combustion Science, vol. 35, no. 1, pp. 57-97, 2009.

[2] C. Pera, S. Chevillard, and J. Reveillon, "Effects of residual burnt gas heterogeneity on early flame propagation and on cyclic variability in spark-ignited engines," Combustion and Flame, vol. 160, no. 6, pp. 1020-1032, 2013.

[3] N. Swaminathan, R. Grout, and E. Mastorakos, "Direct simulations of forced ignition in stratified mixture," tech. rep., European Combustion Meeting, 2007.

[4] N. Peters, Turbulent combustion. Cambridge university press, 2000.

[5] D. Patel and N. Chakraborty, "Effects of mixture distribution on localized forced ignition of stratified mixtures: A direct numerical simulation study," Combustion Science and Technology, vol. 188, no. 11-12, pp. 1904-1924, 2016.

[6] E. Fernandez-Tarrazo, A. L. Sanchez, A. Linan, and F. A. Williams, "A simple one-step chemistry model for partially premixed hydrocarbon combustion," Combustion and Flame, vol. 147, no. 1-2, pp. 32-38, 2006.

[7] D. Patel and N. Chakraborty, "Effects of energy deposition characteristics on localized forced ignition of homogeneous mixtures," International Journal of Spray and Combustion Dynamics, vol. 7, no. 2, pp. 151-174, 2015.

[8] N. Chakraborty, E. Mastorakos, and R. Cant, "Effects of turbulence on spark ignition in inhomogeneous mixtures: a direct numerical simulation (dns) study," Combustion science and technology, vol. 179, no. 1-2, pp. 293-317, 2007.

[9] V. Eswaran and S. Pope, "Direct numerical simulations of the turbulent mixing of a passive scalar," The Physics of fluids, vol. 31, no. 3, pp. 506-520, 1988.

[10] K. W. Jenkins and R. S. Cant, "Direct numerical simulation of turbulent flame kernels," Recent Advances in DNS and LES, pp. 191-202, 1999.

[11] T. J. Poinsot and S. Lelef, "Boundary conditions for direct simulations of compressible viscous flows," Journal of computational physics, vol. 101, no. 1, pp. 104-129, 1992.

[12] R. S. Rogallo, "Numerical experiments in homogeneous turbulence," tech. rep., NASA Ames Research Center; Moffett Field, CA, United States, 1981.

[13] J. E. Dec, "Advanced compression-ignition engines-understanding the in-cylinder processes," Proceedings of the combustion institute, vol. 32, no. 2, pp. 2727-2742, 2009.

[14] B. Renou, E. Samson, A. Boukhalfa, "An experimental study of freely propagating turbulent propane/air flames in stratified inhomogeneous mixtures," Combustion science and technology, no. 11, 2004. 